\title{
475221 - ONLINE TEACHING AID FOR THE 20 STANDARD DIAGNOSTIC TEE VIEWS
}

\author{
Annette Vegas, MD, FRCPC, Massimiliano Meineri, MD, Michael Corrin, MSc, \\ BMC, Gordon Tait, PhD \\ Anesthesia, Toronto General Hospital, Toronto, ON, Canada
}

Introduction: The scope of transesophageal echocardiography (TEE) is increasing as it is becoming a standard imaging tool in the intensive care unit, and intraoperatively in cardiac and non cardiac surgery. Anesthesiologists therefore have a need for training in conducting and interpreting a TEE exam. To assist with this training, we have created a new online teaching aid for TEE and incorporated it in a Web site. The primary challenge in learning TEE is translating the two dimensional (2D) echocardiographic image into a visualization of the three-dimensional (3D) structure of the heart. This Web site provides a learning environment where users can view all of the 20 standard TEE positions with a $3 \mathrm{D}$ heart model showing a representation of the plane of the echocardiographic image. The student is able to rotate the 3-D heart model and echo plane to view it from any perspective, helping them to relate the echocardiographic image to the structures of the heart. Users are also able to remove the part of the 3D image above the echo plane, revealing the internal structures of the heart that correspond to the TEE image in the video clip of that view as shown in Figure 1. This resource can be used by educators for teaching small group sessions and by students for self-study.

Methods: A digital 3D model of the heart was constructed that provides an accurate representation of the exterior and interior structures of the heart. The 3D model allowed sections of the heart to be created along the plane of the TEE image. The cross section revealed when the portion of the model above the echo plane is removed represents the structures seen in the TEE image. Iterative adjustment of the 3D model was used until there was a good match between the video images for all 20 standard diagnostic TEE views and the corresponding cross sections of the model. The TEE images thus acted as a "reality check" on the 3D model, ensuring its accuracy.

Results: Figure 1 shows the 3D heart model on the left with the portion above the echo plane removed for the mid esophageal right ventricle inflow/outflow view, and the corresponding TEE video clip on the right. Face and content validity was assessed with 3 staff anesthesiologists and 2 cardiologists, and usability was assessed using 5 anesthesia residents. Iterative changes to the model and the navigation were based on this evaluation. Construct validity will be carried out with anesthesia and cardiology Fellows, assessing their improvement in knowledge of the 20 standard views with a pre-test/posttest design.

Discussion: This free online teaching aid will provide a unique resource that will be valuable for both educators and trainees learning to interpret TEE images nationally and around the world.

References: None 

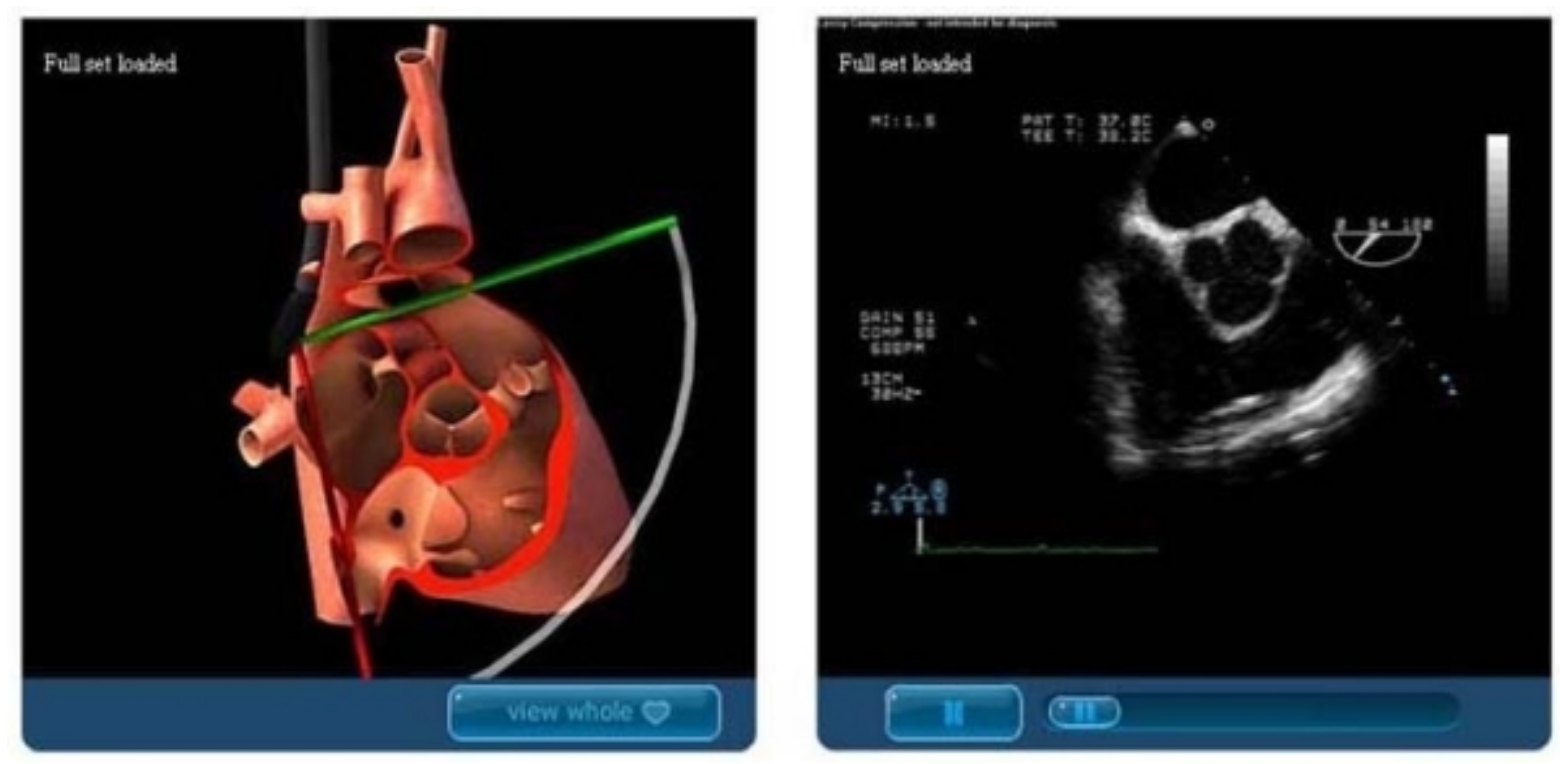